\section{A puzzlling case of cryptococcal meningitis}

To the Editor: We recently admitted a young immunocompetent man with cryptococcal meningitis. He presented alone, and a combination of language barrier and blunted cerebral function hampered history taking. He described 1 week of headache and fever, and gave a vague account of a penetrating head injury 6 months previously.

It was difficult to explain why this otherwise healthy young man, with no evident risk factors for poor T-cell function, had encapsulated yeasts growing in his cerebrospinal fluid. Multiple HIV rapid antigen tests were negative, and 2 weeks of intravenous amphotericin B and oral fluconazole did little to improve his condition.

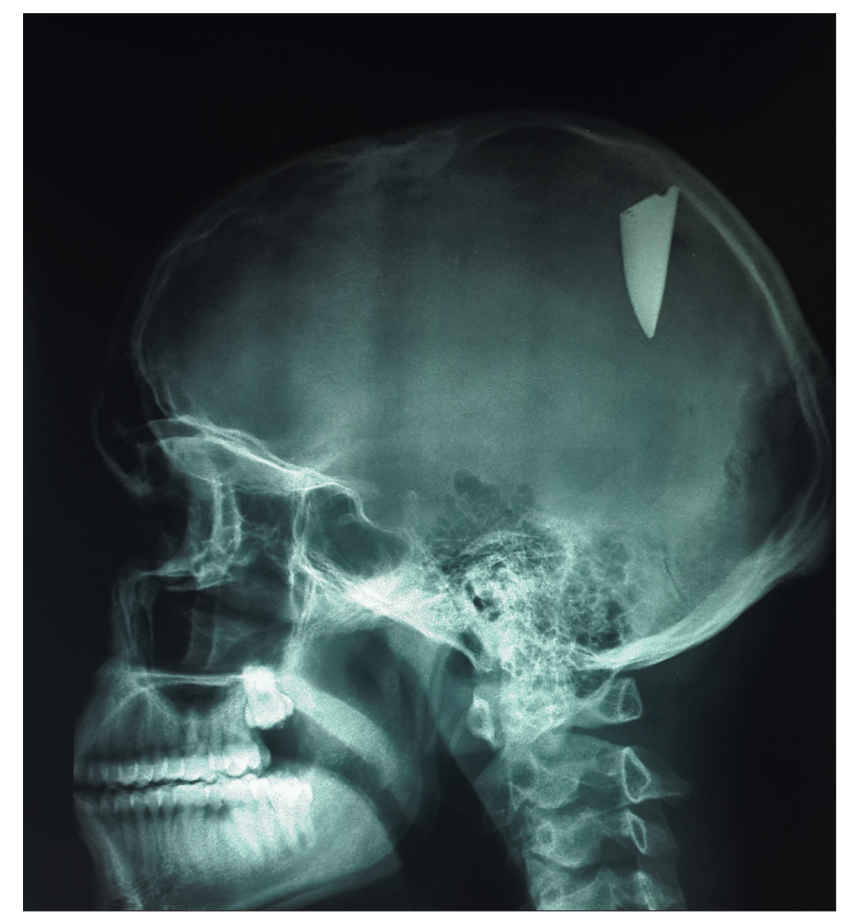

Fig. 1. Cryptococcal meningitis in an otherwise healthy young man - the puzzle solved.

We were poised to embark on the somewhat lengthy referral procedure for a computed tomography brain scan at our tertiary centre when our patient noticed a small amount of pus discharging from a scar on his scalp. A firm prominence was palpated just under the scar, and a subsequent X-ray solved the mystery (Fig. 1).

After surgical removal of the knife blade, the meningitis resolved within several days. The patient was then able to give a more detailed history, and it transpired that he had not come to hospital after the initial injury 6 months earlier because of transport and financial constraints.

A retained foreign body is an often-overlooked differential diagnosis in patients who present with atypical infection. ${ }^{[1]}$ A good history is the single most useful tool in making the diagnosis. This has been well described in the context of inhaled objects in the paediatric population..$^{[2]}$

Difficulty in obtaining a complete history can delay diagnosis and definitive treatment. Maintaining a high index of suspicion, and early use of simple imaging where there is any possibility of prior penetrating trauma, may assist in early exclusion of a retained foreign body. Making a delayed diagnosis by means of unnecessary and expensive investigations at tertiary referral centres can then be avoided.

\section{Stewart James Brown \\ Simon George}

\section{Kate Braithwaite}

Tintswalo Hospital, Acornhoek, Mpumalanga, South Africa simongeorge@hotmail.co.uk

\footnotetext{
1. Anderson MA, Newmeyer WL 3rd, Kilgore ES Jr. Diagnosis and treatment of retained foreign bodies in the hand. Am J Surg 1982;144(1):63-67.

2. Hilliard T, Sim R, Saunders M, Hewer SL, Henderson J. Delayed diagnosis of foreign body aspiration in children. Emerg Med J 2003;20(1):100-101. [http://dx.doi.org/10.1136/emj.20.1.100]
}

S Afr Med J 2014;104(11):720. DOI:10.7196/SAMJ.8804 